\title{
MULTISCALE FE-MD COUPLING: INFLUENCE OF THE CHAIN LENGTH ON THE MECHANICAL BEHAVIOR OF COARSE-GRAINED POLYSTYRENE
}

\author{
Maximilian Ries ${ }^{1}$, Felix Weber ${ }^{2}$, Maximilian Striegel ${ }^{3}$, Paul Steinmann ${ }^{4}$ and Sebastian \\ Pfaller $^{5}$
}

\author{
Institute of Applied Mechanics \\ Friedrich-Alexander University Erlangen-Nürnberg \\ Egerlandstr. 5, 91058 Erlangen, Germany \\ ${ }^{1}$ maximilian.ries@fau.de \\ ${ }^{2}$ felix.w.weber@fau.de \\ ${ }^{3}$ maximilian.striegel@fau.de \\ ${ }^{4}$ paul.steinmann@fau.de \\ ${ }^{5}$ sebastian.pfaller@fau.de
}

Key words: coarse graining, multiscale-modeling, mechanical behavior, molecular dynamics

\begin{abstract}
Polymers have become increasingly essential to cope with modern engineering challenges. To better understand their microstructure's influence, multi-scale approaches that couple molecular dynamics and continuum mechanics are emerging progressively. However, these simulation techniques require detailed knowledge of the material behavior, which is commonly derived from molecular dynamics simulations. Reducing the degrees of freedom by coarse graining enables the investigation of sufficiently large samples and thus we focus on coarse-grained (CG) polystyrene as a model material.

The goal of this contribution is two-fold: Firstly, we identify the minimum sample size necessary to analyze the mechanical properties. Secondly, we quantify the influence of chain length on the material behavior of polystyrene. To this end, we investigate density, end-to-end distance, stress-strain behavior, Young's modulus, and Poisson's ratio. In conclusion, we were able to verify that we can use significantly smaller samples for our investigations without affecting their structure or mechanical behavior. The chain length has a drastic influence on the mechanical properties, with a loss of stiffness in the range of $15 \%$ for very short-chain specimens.
\end{abstract}

\section{INTRODUCTION}

Amorphous polymers, e.g., polystyrene, are used in various applications, but there are still aspects of their mechanical behavior that we do not understand well from an engineering perspective. Phenomena such as the fracture of polymers or polymer composites require a profound knowledge of the underly- 
ing microstructure and its impact on the material's overall properties. Since these effects usually occur on the nano-scale, experiments are often either very demanding or simply impossible. Unfortunately, established numerical methods of different disciplines are not able to solve this problem either: On the one hand, classical continuum approaches rely on a precise constitutive model that cannot fully capture molecular mechanisms. On the other hand, particle-based simulation methods are computationally expensive and limited to examining microscopic specimens insufficient to address engineering problems.

Therefore we combine the advantages of particle-based and continuum methods in a concurrent multiscale approach called the Capriccio method, which is specifically designed to model and investigate amorphous polymers and nanocomposites. The method was introduced by Pfaller et al. $[1,2,3]$ and has been optimized and extended since then $[4,5]$. The Capriccio method has been successfully employed to gain insights into mechanical interphases in polymeric nanocomposites $[6,7]$. It is a partitioned domain approach that couples an atomistic region described by Molecular Dynamics (MD) to a continuum discretized by Finite Elements (FE). In the overlapping region, denoted as the bridging domain (BD), virtual anchor points (APs) are introduced. Each of them is tethered to a specific MD particle via a harmonic spring, and follows the continuum deformation, and thus enables an information transfer between the two domains. The system is solved in the staggered fashion depicted in Fig. 1: A load is applied to the undeformed configuration via the continuum domain resulting in new AP positions. A subsequent equilibration of the particle domain yields anchor point forces due to the connection via harmonic springs. These serve as additional Neumann boundary conditions in the following FE run. These MDFE-iterations are repeated until the deformed system has reached an overall equilibrium.
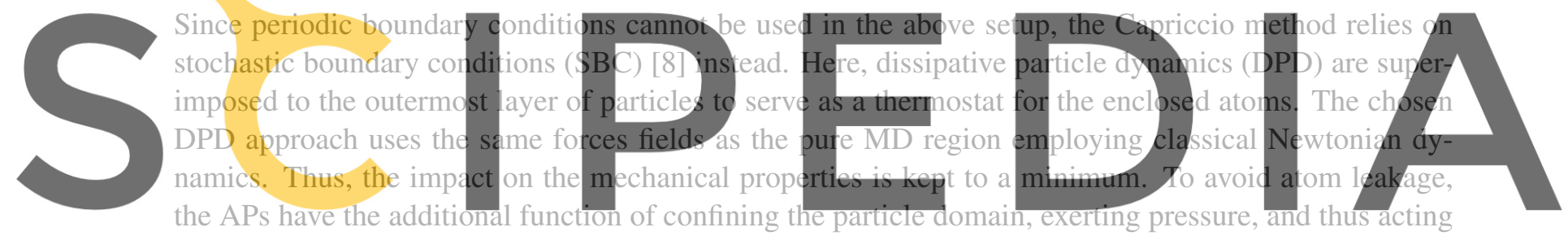

the APs have the additional function of confining the particle domain, exerting pressure, and thus acting
as a barostat. The APs' density increases exponentially towards the border so as to smoothly bridge the

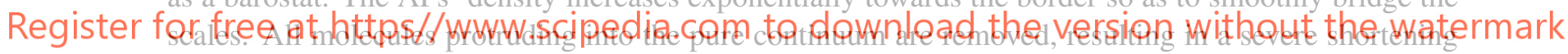

of the polymer chains at the boundary (cf. Fig. 2) and thus causing an unphysical change in the mi-

crostructure. While spatially small, this local change could affect the whole method's performance since the mechanical properties of polymers are known to depend mainly on the molecular structure of the material.

Therefore, in the following, we investigate the extent to which the chain length influences mechanical properties of our polystyrene sample system.

\section{SAMPLE PREPARATION \& UNIAXIAL TENSION SETUP}

In this study, atactic polystyrene serves as a model system and is simulated at a coarse-grained resolution to increase the computational efficiency allowing us to investigate larger systems. Ghanbari et al. [9] used Iterative Boltzmann Inversion [10] to derive appropriate coarse-grained force fields by substituting every styrene monomer with a so-called superatom [11]. The resulting model has already proven its capability and reliability in the following applications: Farah et al. investigated the polymer chain growth [12], Riccardi et al. locally derived the Poisson's ratio [13], Rahimi et al. developed a correlation between 


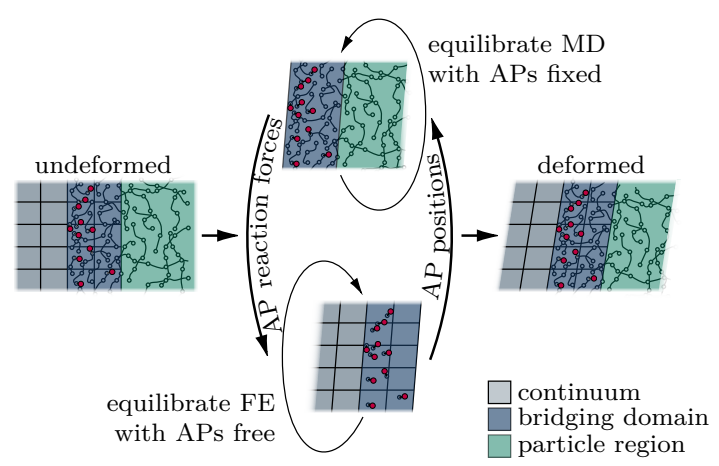

Figure 1: Spatial setup of the Capriccio method: Simulation domain partitioned into continuum, particle region and bridging domain where both descriptions overlap. The coupled solution is obtained in a staggered fashion by carrying out MDFE iterations until an overall equilibrium is reached.

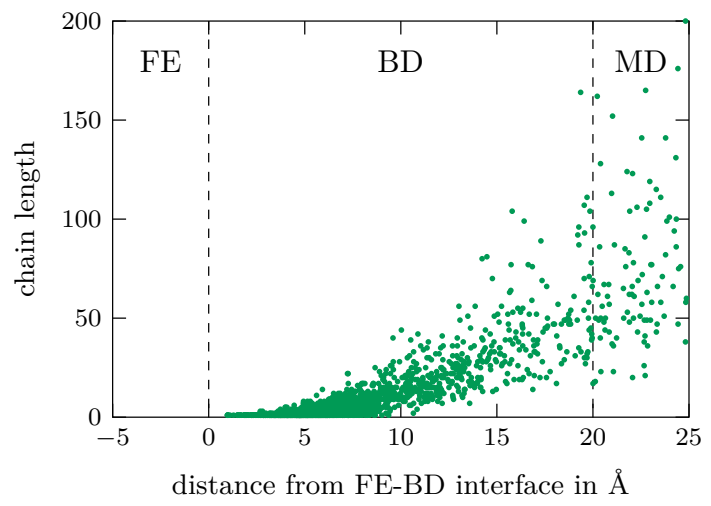

Figure 2: Length of the chains plotted over their center of gravity: The initial chain length of 200 monomers reduces drastically in the bridging domain (BD) due to the cutting of protruding chains required in $\mathrm{SBC}$.

Young's moduli at atomistic and coarse-grained resolution based on the associated glass transition temperatures [16], and Munao et al. carried out a study of nanoparticles in polymer melt [14]. Furthermore, we performed mechanical deformation simulations $[17,18]$ and, on this basis, developed a sophisticated

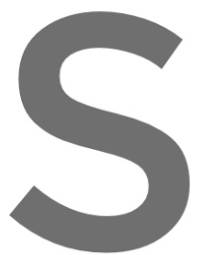
inelastic constitutive law [19].

The polystyrene samples $590 \mathrm{~K}$ and cooled down to induces an implicit speeddiffer from their atomistic
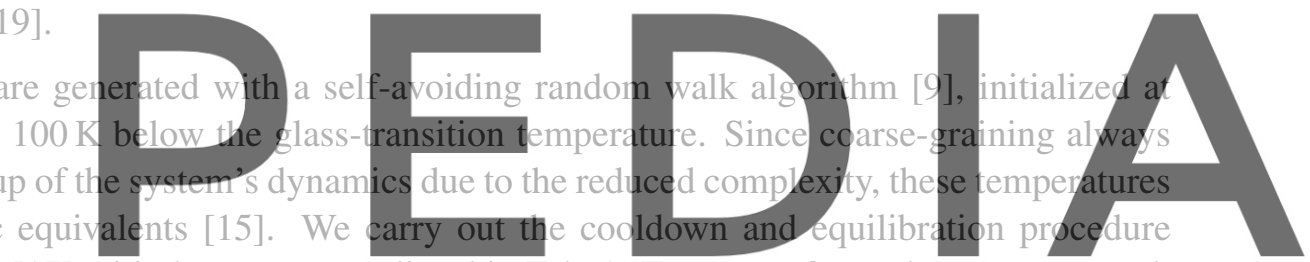

described in more detail in [17] with the parameters listed in Tab. 1. Two sets of samples are generated:

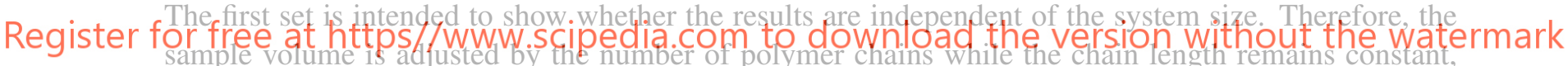

as shown in Tab. 3. The second set aims to investigate the effect of the polymer chain length on the

mechanical properties and contains samples of the same size but with varying chain lengths (cf. Tab. 4). To obtain statistically reliable results, ten randomized variants of each sample type are created and hence all quantities presented hereafter are the corresponding averages.

The uniaxial tension tests are performed analogously to [17]: We deform the samples by mimicking a canonical ensemble in the x-direction, enabling us to set a new box length $l_{x}$. In $y$-and $z$-directions, the atmospheric pressure $p_{\text {atm }}$ is predefined like in an isothermal-isobaric ensemble, allowing for a free lateral contraction, cf. Fig 3. We investigate an overall deformation of $8 \%$ strain, applied in load steps of $\Delta \varepsilon_{x x}=0.1 \%$ where $\Delta n=40000$ time steps are evaluated in each of these steps. In order to achieve strain rates $\dot{\varepsilon}_{x x}$ of $0.1 \% \mathrm{~ns}^{-1}, 1 \% \mathrm{~ns}^{-1}$, and $20 \% \mathrm{~ns}^{-1}$ the time step is adjusted according to

$$
\Delta t=\frac{\Delta \varepsilon_{x x}}{\Delta n \dot{\varepsilon}_{x x}}
$$

and listed together with the remaining parameters in Tab. 2. 
Table 1: MD parameters for the cooldown simulations.

\begin{tabular}{ll}
\hline time step & $5 \mathrm{fs}$ \\
temperature coupling time & $200 \mathrm{fs}$ \\
pressure coupling time & $500 \mathrm{fs}$ \\
isothermal compressibility & $1 \times 10^{-6} \mathrm{kPa}$ \\
atmospheric pressure & $101.3 \mathrm{kPa}$ \\
cooling rate & $5 \mathrm{Kns}^{-1}$ \\
\hline
\end{tabular}

Table 2: MD parameters for the uniaxial tension tests.

\begin{tabular}{ll}
\hline maximum strain & $8 \%$ \\
strain increment & $0.1 \%$ \\
strain rates & $0.1,1,20 \% \mathrm{~ns}^{-1}$ \\
time steps per load step & 40000 \\
time step & $25,2.5,0.125 \mathrm{fs}$ \\
temperature & $100 \mathrm{~K}$ \\
isothermal compressibility & $1 \times 10^{-6} \mathrm{kPa}$ \\
atmospheric pressure & $101.3 \mathrm{kPa}$ \\
temperature coupling time & $200 \mathrm{fs}$ \\
pressure coupling time & $5000 \mathrm{fs}$ \\
\hline
\end{tabular}

All simulations are carried out with the MD software IBIsCO specifically developed for coarse-grained force fields [15].

Table 3: Set of samples to identify size effect. num. of chains chain length tot. num. of atoms

Table 4: Set of samples to investigate effect of chain length.

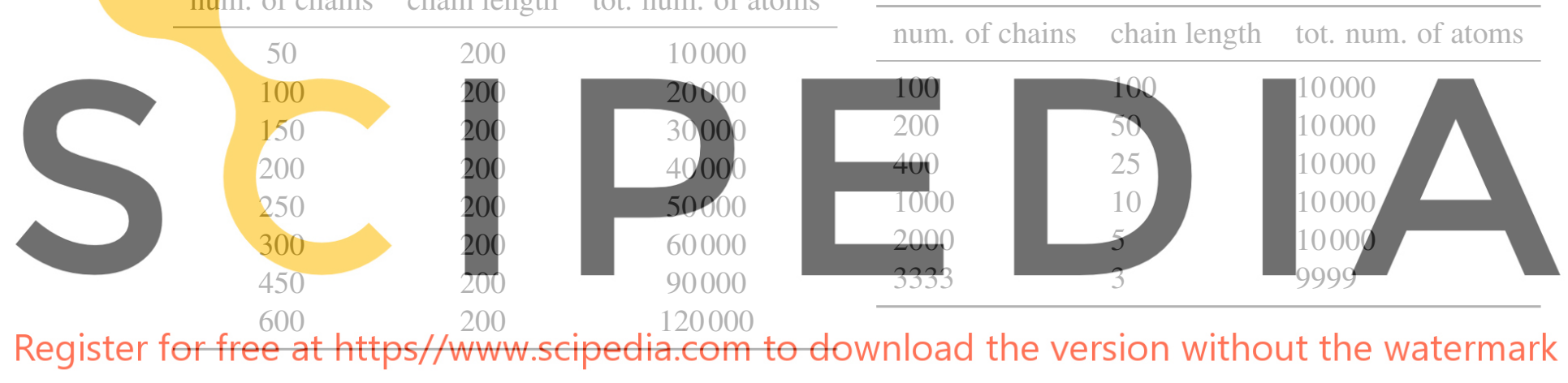

\section{SAMPLE SIZE}

Since the necessary computing time of our MD simulations scales linearly with the number of atoms, we strive to identify the minimum sample size to reduce the computational costs. Therefore, we compare structural quantities such as density and end-to-end distance and mechanical quantities such as stress, Young's modulus, and Poisson's ratio over a wide range of sample sizes (cf. Tab. 3).

After the cooldown, the resulting densities, averaged over the ten variations mentioned above, are depicted in Fig. 4. Samples with fewer than 40000 atoms are marginally denser, but the deviation relative to the largest system is as small as $0.2 \%$ and is therefore considered insignificant. In contrast, the smallest sample's standard deviation is more than twice that of the largest, which can be attributed to the comparatively larger impact of local anomalies. This becomes visible by comparing the density distributions of two individual systems as shown in Fig. 6: While the large sample features a nearly homogeneous distribution, the small system exhibits local spots of significantly higher or lower density.

Another essential characteristic of thermoplastics' structure is the end-to-end distance of the individual 


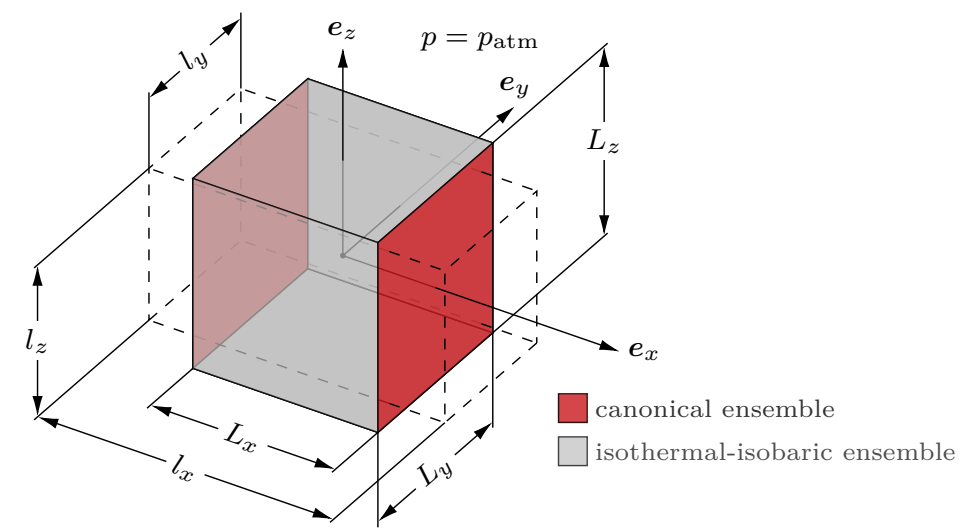

Figure 3: MD setup for uniaxial deformation in $x$-direction: Deformation applied via canonical ensemble in tensile direction and atmospheric pressure $p_{\text {atm }}$ imposed with isothermal-isobaric ensemble in lateral directions.

\section{polymer chains, which is shown in Fig. 5 for different system sizes. In the same vein as the density,} the end-to-end distance remains at the same level, but a significantly higher standard deviation can be observed for smaller systems.
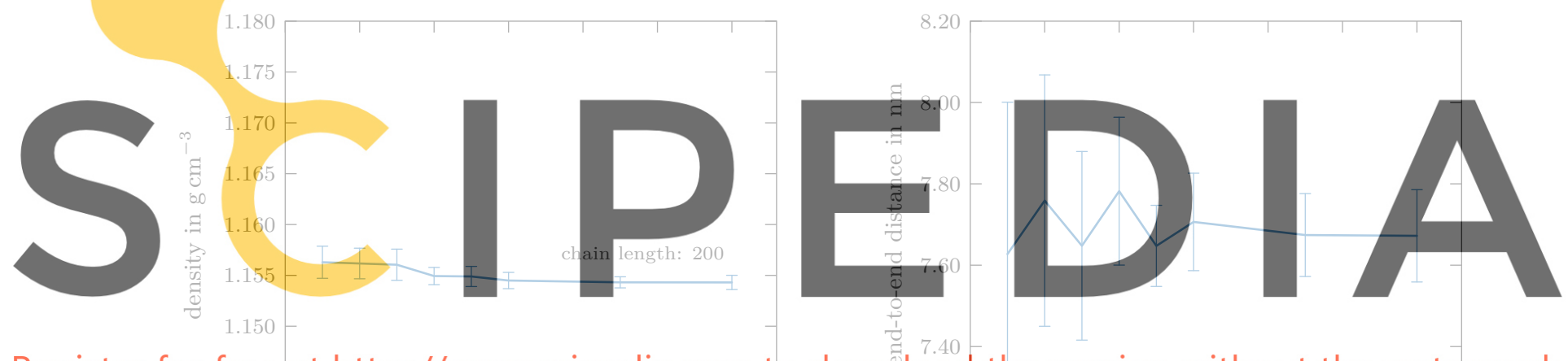

Register for free at https//www.scipedia.com to
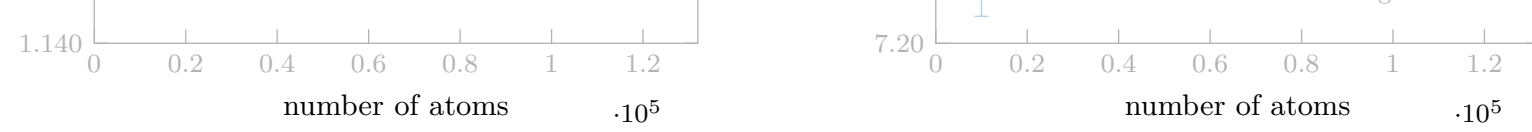

Figure 4: Resulting mass density after cooldown and equilibration for varying sample size with chain length of 200, averaged over 10 samples, standard deviation indicated by error bars.

Figure 5: Resulting end-to-end distance after cooldown and equilibration for varying sample size with chain length of 200, averaged over 10 samples, standard deviation indicated by error bars.

Next, we want to clarify whether the system size influences the mechanical properties of the polymer. For this purpose, we perform uniaxial tension tests under various constant strain rates. We evaluate the virial stress tensor as introduced by Thompson et. al [20]. Since the deformation is applied in discrete load steps, the stress obtained is averaged over the last fifth of each load step as suggested in [17]. In this way, stress peaks that arise inevitably due to the non-continuous loading are filtered out. From the resulting stress-strain relations for different sample sizes shown in Fig. 7 two observations can be made: First, higher strain rates lead to higher stresses which is coherent with previous investigations [17]. Second, 

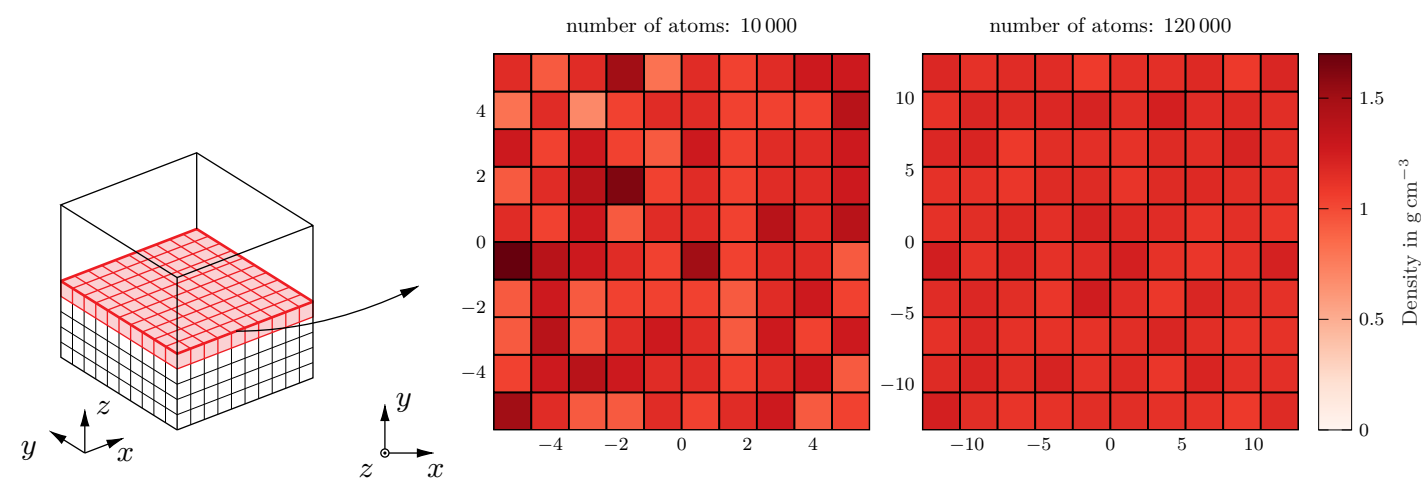

Figure 6: Density distribution computed voxel-wise and plotted for highlighted mid-plane for smallest and largest sample, both with chain length of 200 .

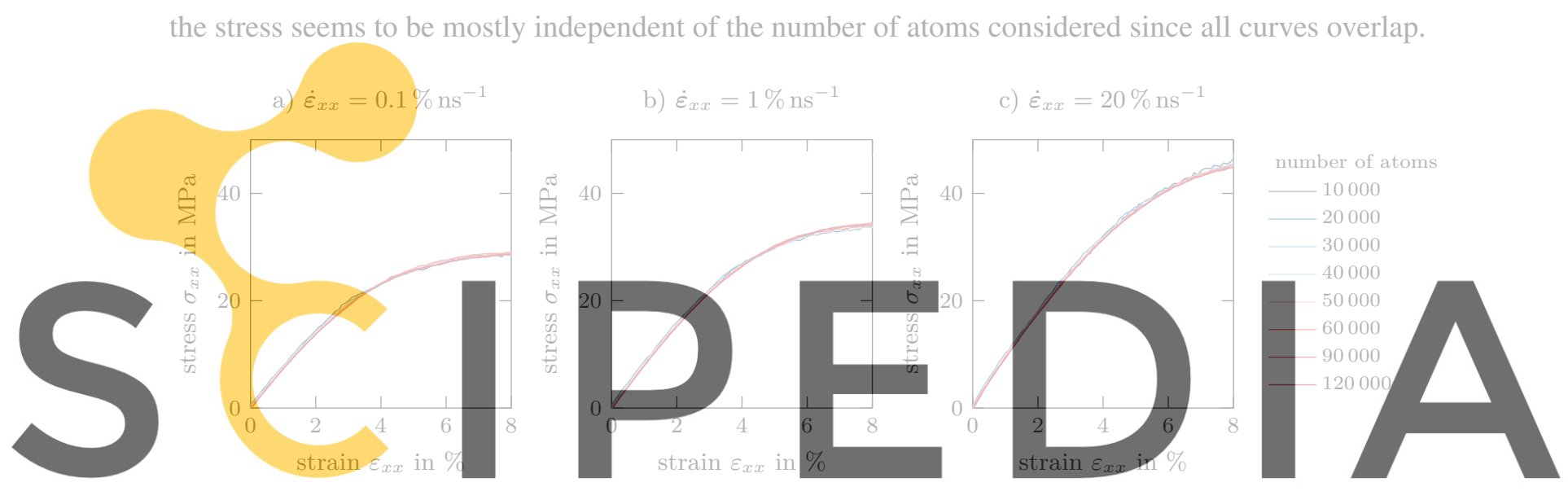

Figure 7: Resulting stress-strain relation for various sample sizes and 200 monomers per chain evaluated at strain

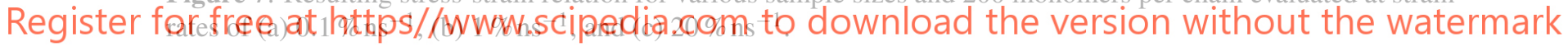

To investigate this further, we calculate the effective Young's modulus as the slope of the secant of the stress between $\varepsilon_{x x}=0 \%$ and $1 \%$. The result is given in Fig. 8 and underlines the previous statements. The polystyrene tested here behaves stiffer under higher strain rates, but the associated Young's moduli seem to be independent of the system size. However, the standard deviation increases significantly with decreasing sample size, most likely due to the more pronounced variations in the initial configurations discussed above (cf. Fig. 4 and Fig. 5). The derived Young's moduli are in good agreement with previous studies [16, 17], but are significantly smaller than observed in experiments due to the coarse-graining [3].

Since the polymer behaves isotropic $\left(\varepsilon_{y y} \approx \varepsilon_{z z}\right)$ we calculate the Poisson's ratio as $\mathrm{v}=-\frac{\varepsilon_{y y}}{\varepsilon_{x x}}$ visualized in Fig. 9. Due to the system's inertia, the $v$ starts close to 0 and steeply rises until it transitions to a linear increase at about $2 \%$ strain. Higher strain rates cause lower Poisson's ratio as well as significantly fewer fluctuations and thus smoother curves. Although the variously sized systems' results differ, no clear trend can be derived, but smaller systems are in general subject to larger fluctuations.

In summary, the effect of the sample size on the structure (density and end-to-end distance) and mechan- 


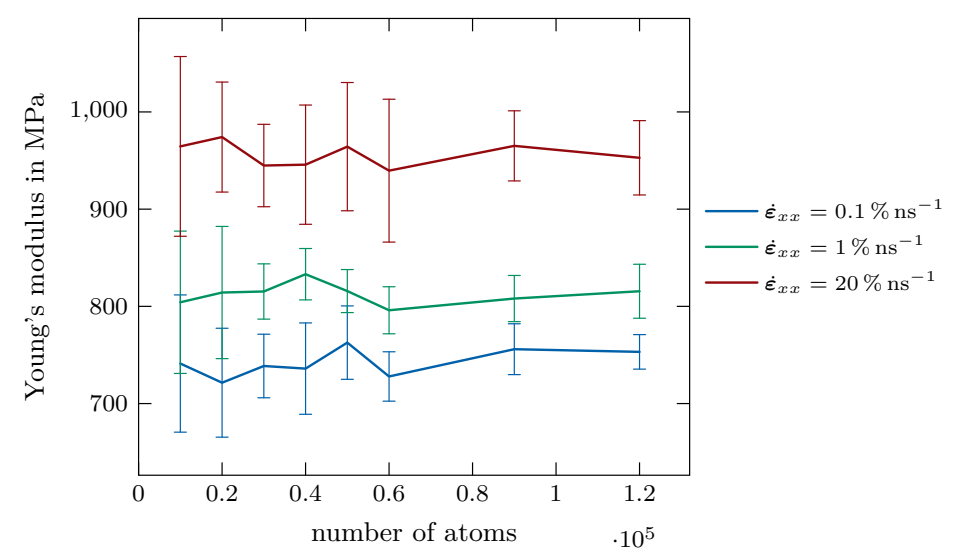

Figure 8: Resulting Young's modulus for various sample sizes and 200 monomers per chain evaluated at strain rates of $0.1 \% \mathrm{~ns}^{-1}, 1 \% \mathrm{~ns}^{-1}$, and $20 \% \mathrm{~ns}^{-1}$, standard deviation indicated by error bars.

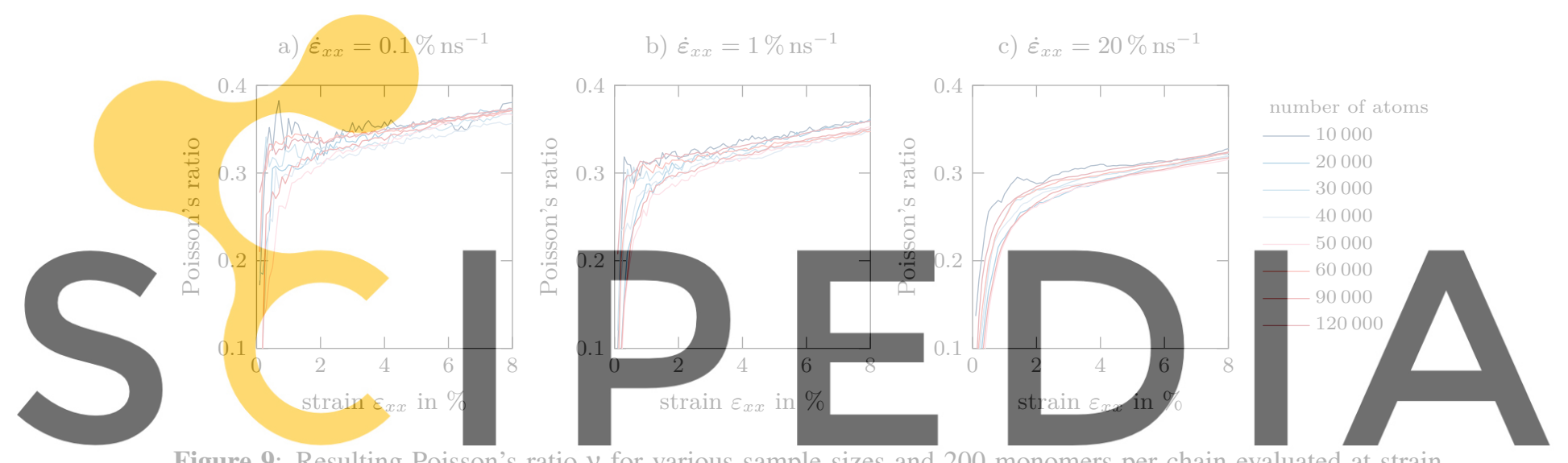

Figure 9: Resulting Poisson's ratio $v$ for various sample sizes and 200 monomers per chain evaluated at strain

Register for free at https//www.scipedia.com to download the version without the watermark

ical behavior (stress, Young's modulus, Poisson's ratio) is negligible. However, the variability of these quantities increases with a decreasing number of atoms, limiting the reliability of the derived statements. Consequently, we conduct the variation of the chain length with the smallest systems investigated in this section, i.e., 10000 atoms. We do not shrink the samples any further since local peculiarities would increasingly determine the system characteristics, and reliable statements could no longer be made.

\section{CHAIN LENGTH}

After clarifying the minimum size of our samples, we can now address the second aim of our investigation: Evaluating the influence of the chain length on the structure and mechanical properties of the samples. As before, we first consider the resulting average density, which is shown in Fig. 10. Starting from $1.16 \mathrm{~g} \mathrm{~cm}^{-3}$ at a chain length of 200 , it decreases slowly at first and then falls sharply for chain lengths below 50 down to a value of $1.00 \mathrm{~g} \mathrm{~cm}^{-3}$ at a chain length of 3 . As expected, the end-to-end distance reduces with decreasing chain length, but in a nonlinear manner (cf. Fig. 11). Its standard deviation approaches zero for very short chains, indicating that these short molecules tend to translate rather 
than deform.

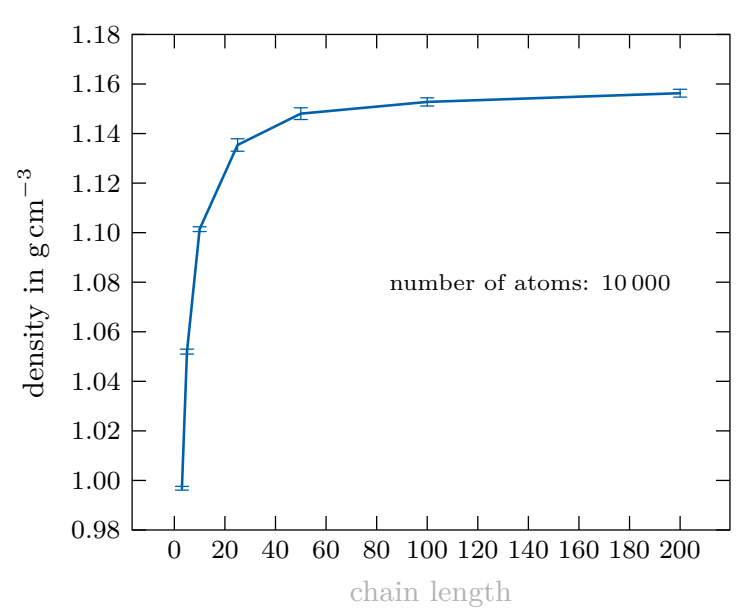

Figure 10: Resulting mass density after cooldown and equilibration for varying chain lengths and a sample size of 10000 atoms, averaged over 10 samples, standard deviation indicated by error bars.

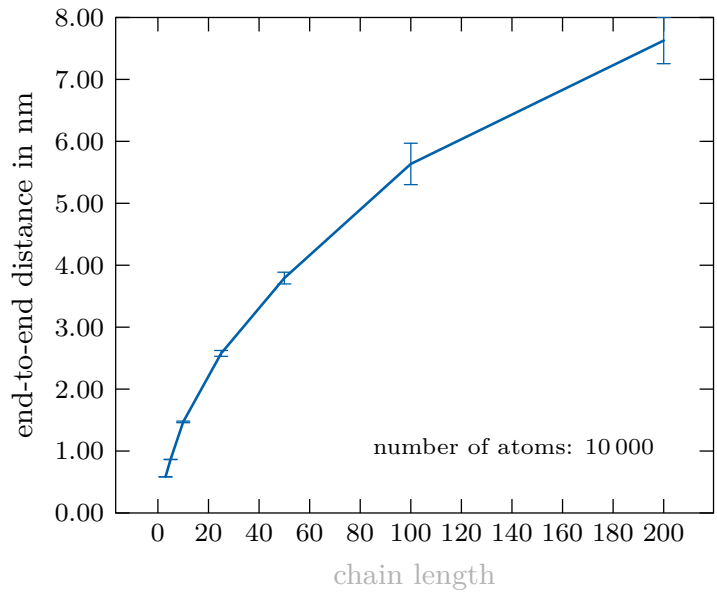

Figure 11: Resulting end-to-end distance after cooldown and equilibration for varying chain lengths and a sample size of 10000 atoms, averaged over 10 samples, standard deviation indicated by error bars.

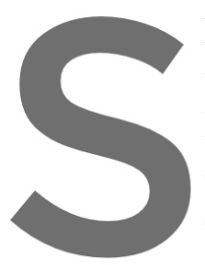

Fig. 12 displays the stres

thermore, the short-chain

lengths below 25. This lo

density, as less dense polymers also tend to be soft

entanglements essential fo
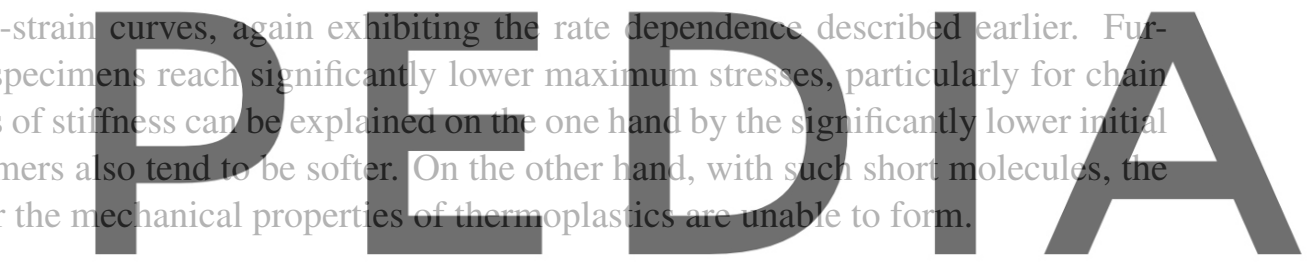

a) $\dot{\varepsilon}_{x x}=0.1 \% \mathrm{~ns}^{-1}$

b) $\dot{\varepsilon}_{x x}=1 \% \mathrm{~ns}^{-}$

c) $\dot{\varepsilon}_{x x}=20 \% \mathrm{~ns}^{-}$

Register for free at https//www.scipedia.com to download the version without the watermark
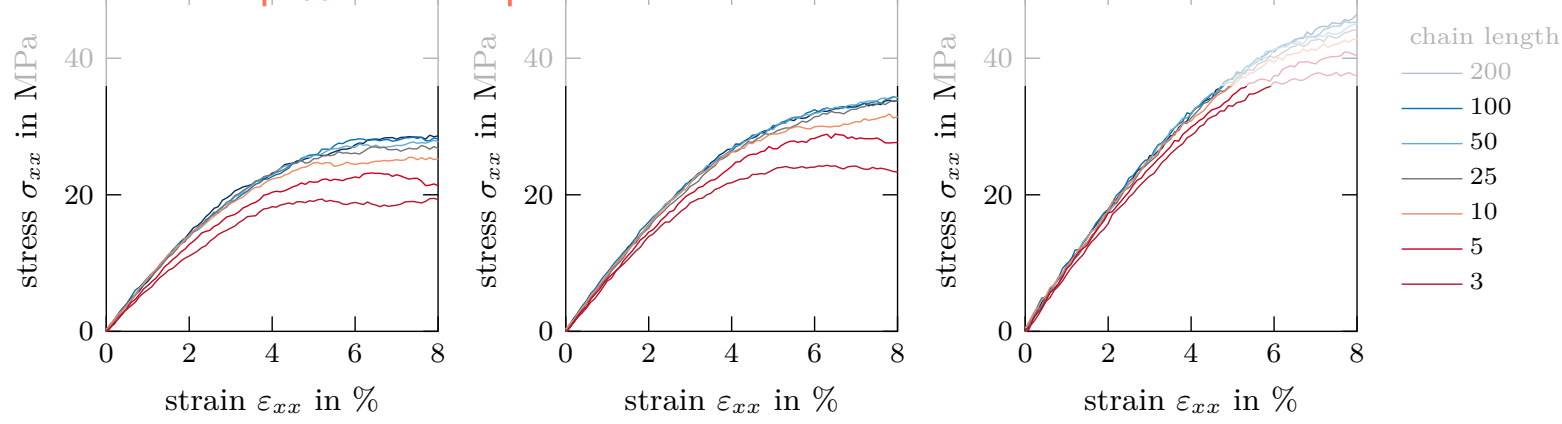

Figure 12: Resulting stress-strain relation for various chain lengths and a sample size of 10000 atoms, evaluated at strain rates of (a) $0.1 \% \mathrm{~ns}^{-1}$, (b) $1 \% \mathrm{~ns}^{-1}$, and (c) $20 \% \mathrm{~ns}^{-1}$.

Quantitatively, this is best expressed by Young's modulus depicted in Fig. 13, which is subject to a high variability due to the small sample size. Nevertheless, there is a clear tendency that shorter chains lead to a reduced Young's modulus. The stiffness loss of the specimens with the shortest chains relative to 
the longest ones amounts to $18 \%, 12 \%$, and $15 \%$ at strain rates of $0.1 \% \mathrm{~ns}^{-1}, 1 \% \mathrm{~ns}^{-1}$, and $20 \% \mathrm{~ns}^{-1}$, respectively.

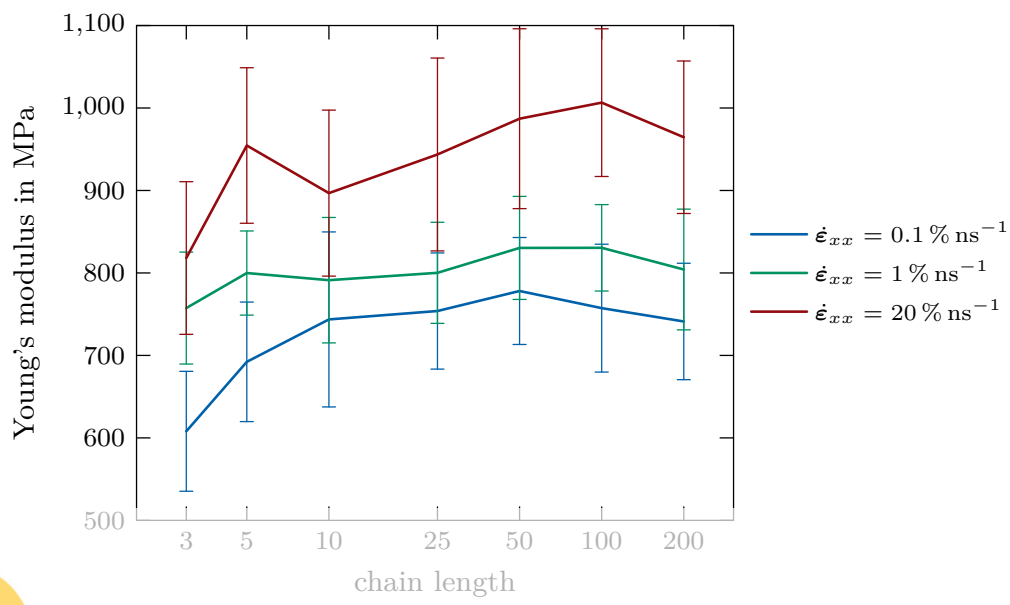

Figure 13: Resulting Young's modulus for various chain lengths and a sample size of 10000 atoms, evaluated at strain rates of $0.1 \% \mathrm{~ns}^{-1}, 1 \% \mathrm{~ns}^{-1}$, and $20 \% \mathrm{~ns}^{-1}$, standard deviation indicated by error bars.

Finally, Fig. 14 shows the Poisson's ratio for different chain lengths and strain rates. As before, v exhibits an initial build up to about $2 \%$ strain and-increases linearly from there. Peculiarly, for strain rates of $0.1 \% \mathrm{~ns}^{-1}$ and 1 initial Poisson's ratios gccur Also for the linearly increasing range above $2 \%$ strain, no clear trend related to the identified, but the differences between long- and short smaller strain rates.

Register for freeatthtes/\%wWw.scipedia.com to download the versiom\%without the watermark
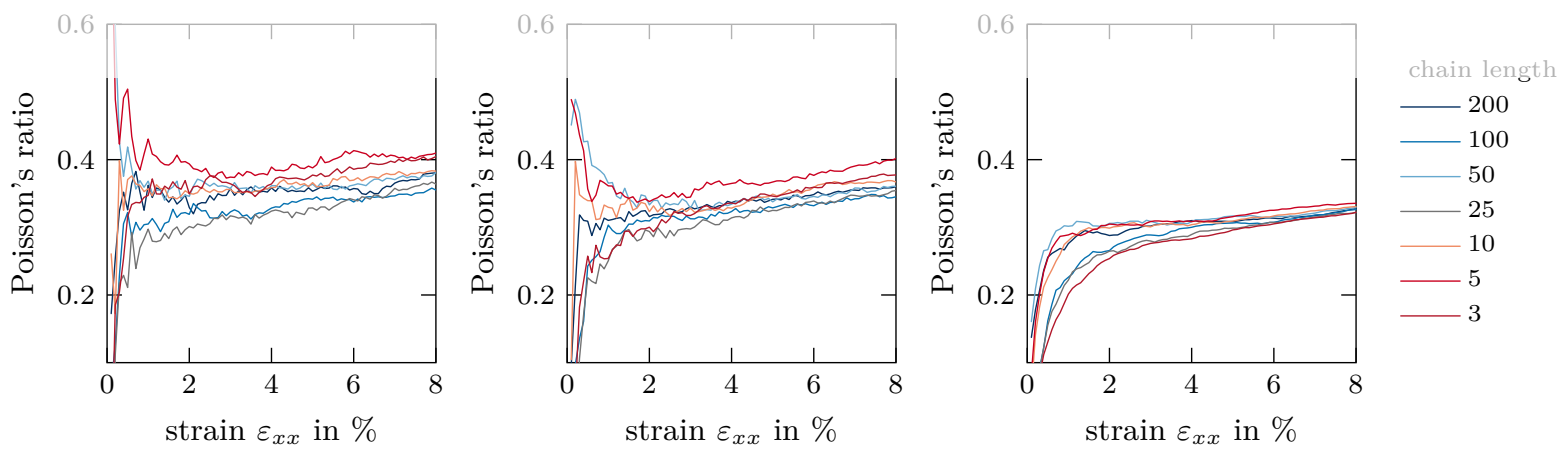

Figure 14: Resulting Poisson's ratio $v$ for various chain lengths and a sample size of 10000 atoms, evaluated at strain rates of (a) $0.1 \% \mathrm{~ns}^{-1}$, (b) $1 \% \mathrm{~ns}^{-1}$, and (c) $20 \% \mathrm{~ns}^{-1}$. 


\section{CONCLUSION}

In conclusion, we were able to verify that we can use significantly smaller samples for our investigations without affecting their structure or mechanical behavior, i.e. there is no size effect. This reduces the computational costs significantly at the expense of a larger variability of the derived results. The second part of this investigation has revealed that shorter chains have immense effect on the structural properties, resulting in a decrease of the density. The mechanical properties are also drastically affected, with a loss of stiffness in the range of $15 \%$ for very short-chain specimens.

Under the stochastic boundary conditions described above, particularly these very short polymer chains investigated here are present due to the chain cutting. The large deviations of the material properties identified in this study are particularly undesirable in the handshake region of a partitioned-domain multiscale approach such as the Capriccio method. This could not only compromise the performance of the coupling scheme but also conceal artifacts of the coupling itself and thus complicate an effective optimization of the method. Consequently, alternative boundary conditions are needed in this context that do not require intervention in the polymer structure.
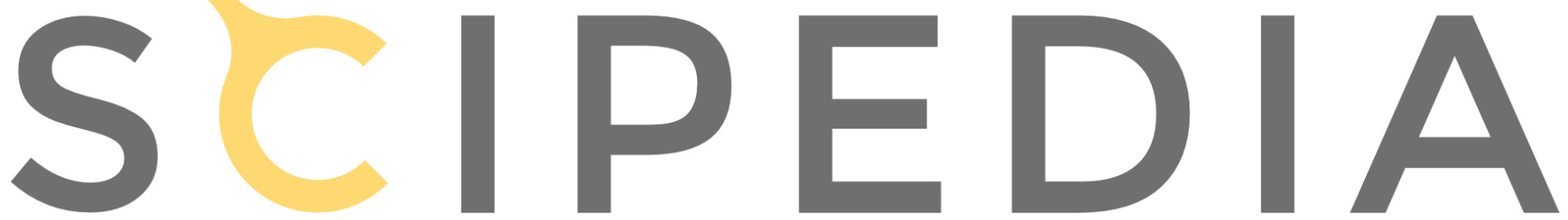

Register for free at https//www.scipedia.com to download the version without the watermark 


\section{REFERENCES}

[1] Pfaller, S.; Possart, G.; Steinmann, P.; Rahimi, M.; Müller-Plathe, F. \& Böhm, M. C. A comparison of staggered solution schemes for coupled particle-continuum systems modeled with the Arlequin method, Computational Mechanics, Springer-Verlag, 2012, 49, 565-579.

[2] Pfaller, S.; Rahimi, M.; Possart, G.; Steinmann, P.; Müller-Plathe, F. \& Böhm, M. An Arlequinbased method to couple molecular dynamics and finite element simulations of amorphous polymers and nanocomposites, Computer Methods in Applied Mechanics and Engineering, 2013, 260, 109129.

[3] Pfaller, S.; Multiscale Simulation of Polymers, Friedrich-Alexander-Universität ErlangenNürnberg, Friedrich-Alexander-Universität Erlangen-Nürnberg, 2015.

[4] Pfaller, S.; Kergaßner, A. \& Steinmann, P.; Optimisation of the Capriccio Method to Couple Particle- and Continuum-Based Simulations of Polymers, Multiscale Science and Engineering, 2019, 1, 318-333.

[5] Zhao, W.; Steinmann, P.; \& Pfaller, S.; A particle-continuum coupling method for multiscale simulations of viscoelastic-viscoplastic amorphous glassy polymers, submitted.

[6] Pfaller, S.; Possart, G.; Steinmann, P.; Rahimi, M.; Müller-Plathe, F. \& Böhm, M.; Investigation of interphase Effects in Silica-Polystyrene Nanocomposites Based on a Hybrid Molecular-DynamicsFinite-Element Simulation Framework, Physical Review E, 2016, 93, 052505.

[7] Ries, M.; Possart, G.; Steinmann, P.; \& Pfaller, S.; A coupled MD-FE methodology to characterize mechanical interphases in polymeric nanocomposites, in preparation.

[8] Rahimi, M.; Karimi-Varzaneh, H. A.; Böhm, M. C.; Müller-Plathe, F.; Pfaller, S.; Possart, G. \& Steinmann, P. Nonperiodic stochastic boundary conditions for molecular dynamics simulations of materials embedded into a continuum mechanics domain, Journal of Chemical Physics, 2011, 134, 154108 .

[9] Ghanbari, A.; Ndoro, T. V. M.; Leroy, F.; Rahimi, M.; Böhm, M. C. \& Müller-Plathe, F. Interphase Structure in Silica-Polystyrene Nanocomposites: A Coarse-Grained Molecular Dynamics Study, Macromolecules, 2012, 45, 572-584.

[10] Reith, D.; Pütz, M. \& Müller-Plathe, F.; Deriving effective mesoscale potentials from atomistic simulations, Journal of Computational Chemistry, 2003, 24, 1624-1636.

[11] Qian, H.; Carbone, P.; Chen, X.; Karimi-Varzaneh, H. A. \& Müller-Plathe, F.; TemperatureTransferable Coarse-Grained Potentials for Ethylbenzene, Polystyrene, and Their Mixtures, Macromolecules, 2008, 41, 9919-9929.

[12] Farah, K.; Karimi-Varzaneh, H. A.; Müller-Plathe, F. \& Böhm, M. C.; Reactive molecular dynamics with material-specific coarse-grained potentials: growth of polystyrene chains from styrene monomers, The Journal of Physical Chemistry B, ACS Publications, 2010, 114, 13656-13666.

[13] Riccardi, E.; Böhm, M. C. \& Müller-Plathe, F.; Molecular dynamics method to locally resolve Poisson's ratio: Mechanical description of the solid-soft-matter interphase, Phys. Rev. E, American Physical Society, 2012, 86, 036704. 
[14] Munaò, G.; Pizzirusso, A.; Kalogirou, A.; De Nicola, A.; Kawakatsu, T.; Müller-Plathe, F. \& Milano, G.; Molecular structure and multi-body potential of mean force in silica-polystyrene nanocomposites, Nanoscale, Royal Society of Chemistry, 2018, 10, 21656-21670.

[15] Karimi-Varzaneh, H. A.; van der Vegt, N. F.; Müller-Plathe, F. \& Carbone, P.; How good are coarse-grained polymer models? A comparison for atactic polystyrene, ChemPhysChem, Wiley Online Library, 2012, 13, 3428-3439.

[16] Rahimi, M.; Iriarte-Carretero, I.; Ghanbari, A.; Böhm, M. C. \& Müller-Plathe, F.; Mechanical behavior and interphase structure in a silica-polystyrene nanocomposite under uniaxial deformation, Nanotechnology, 2012, 23, 305702.

[17] Ries, M.; Possart, G.; Steinmann, P. \& Pfaller, S.; Extensive CGMD Simulations of Atactic PS Providing Pseudo Experimental Data to Calibrate Nonlinear Inelastic Continuum Mechanical Constitutive Laws, Polymers, Multidisciplinary Digital Publishing Institute, 2019, 11, 1824.

[18] Ries, M.; Steinmann, P. \& Pfaller, S.; Characterization of Polystyrene under Shear Deformation Using Molecular Dynamics, Developments and Novel Approaches in Nonlinear Solid Body Mechanics, Springer, 2020, 219-229.

[19] Zhao, W.; Ries, M.; Steinmann, P. \& Pfaller, S.; A viscoelastic constitutive model for polymers based on molecular dynamics simulation under finite uniaxial deformation, submitted.

[20] A. P. Thompson, S. J. Plimpton, \& W. Mattson. ; General formulation of pressure and stress tensor for arbitrary many-body interaction potentials under periodic boundary conditions, The Journal of chemical physics, 2009, 131.15, 154107. 